\title{
Editorial
}

\section{Medical humanities}

David Greaves and Martyn Evans Editors, Medical Humanities, Centre for Philosophy and Health Care, University of Wales Swansea

In Britain interest in "medical humanities" has emerged only recently, whereas in the United States it has been developing over the past thirty years. What then has generated this concern and why now? The answer seems to be that it is a second generational response to the shortcomings of a medical culture dominated by scientific, technical and managerial approaches. The first response came in the 1960s and 1970s and led in Britain to the emergence of medical sociology, social history of medicine and medical ethics as academically respectable subdisciplines. The current response can then be seen as a new phase in which medical humanities is making its first appearance in Britain, and is also taking a new and related direction in North America.

Within this overall picture two main formulations of medical humanities are apparent. The first is concerned with complementing medical science and technology through the contrasting perspective of the arts and humanities, but without either side impinging on the other. The second aims to refocus the whole of medicine in relation to an understanding of what it is to be fully human; the reuniting of technical and humanistic knowledge and practice is central to this enterprise. We have described these two approaches to medical humanities as "additive" and "integrated" respectively ${ }^{1}$ and Richard Smith summed them up as follows:

"The additive view is that medicine can be 'softened' by exposing its practitioners to the humanities; the integrated view is more ambitious, aiming to shape the "nature, goals and knowledge base' of medicine itself'."’

In reality there is a wide spectrum of literature emerging which lies on a continuum between these two positions, and collectively it reflects an ongoing debate as to the best way forward. The aim of the new journal, Medical Humanities, will be to promote all aspects of this debate. It will aim also to avoid merely fostering a further series of discrete medical subdisciplines relating to various of the arts. Although those subdisciplines might have their own internal validity, causing them to proliferate would be against the spirit of the wider enterprise of the integrated conception of medical humanities. Indeed, seen in this broader perspective medical humanities does not fit the traditional notion of disciplines and specialties defined in terms of their knowledge bases, but is better understood as a perspective which gains coherence from its concern with understanding what it is to be "fully human", within a common mode of enquiry.

There is a great deal of literature dispersed throughout a wide range of journals and books which does not appear under the heading of medical humanities, which could well be seen as falling within its scope. Why then establish a new journal when such material is already accommodated elsewhere? First because the issues raised by medical humanities are becoming recognised more widely, and need to be addressed more thoroughly, and above all more coherently, so as to transcend the specific insights generated by individual disciplines. Second because medical ethics is also going through a period of reassessment (partly because of concerns about its role as a subdiscipline of both medicine and applied ethics), which is leading to ethics and humanities in medicine coming closer together in North America. $^{3}$

One way of seeing this is as the gradual emergence of a new viewpoint, in which philosophy and ethics, alongside a whole range of other disciplines, will jointly become reconfigured and so better equipped to address the challenges of contemporary medicine and health care. Medical humanities, in embodying this viewpoint, also requires a strong and distinctive voice which is not currently available in Britain, and the journal aims to fill this niche.

This first issue of Medical Humanities is appropriately of a general nature rather than having a special theme. Essentially the aim is to open up a discussion of the nature and scope of medical 
humanities. Accordingly the first three academic papers in this issue are variously concerned with general matters to do with the identity, role and "operation" of the medical humanities.

Our opening contributor, Anne Scott, considers the relationship between science and the arts, and between clinical practice and the imagination, from her perspective as clinical nurse, nurse teacher and philosopher of nursing. Clearly a central ground for the current interest in the relation between the arts and medicine is the expectation that practitioners can draw insights from the arts and transfer these to their own practice. Scott argues that this is possible only because the arts involve an exploration of what human beings hold in common, as well as what an individual experiences in particular.

The relation between arts and science is at stake too in our second paper. Historian and ethicist Kenneth Boyd is concerned (in the context of an exploration of the meaning of clinical medicine's central concepts) with the relation between the sciences and language, including the imaginative language of metaphor and the religious language of faith. Boyd draws our attention to the pervasive nature of value judgments, images and metaphors in human discourse, such that they extend even into scientific language. Modern understandings of science, argues Boyd, make possible a reconciliation-or at least a mutual respectbetween religious and scientific thought, owing in part to a recognition of the extent to which the imagination is involved in both.

Our third contributor to this "scene-setting" is a physician. John Saunders considers the relation between medical sciences and the humanities via the distinction between empiricist "pure" science as the enquiry after knowledge for its own sake, and "applied" science-including medical science - as the exercise of control over the physical world for human purposes. Saunders commends the fusing of scientific knowledge and the "practical" or "tacit" knowledge by means of which medicine itself becomes an art.

These more general discussions are followed by two papers concerned with the role of literature in medicine, and more specifically in medical education. Jane Macnaughton reviews the development of literature courses in the education of doctors, and accommodates both instrumental and noninstrumental uses of literature as an art form. This distinction is both crucial and controversial, since whilst perhaps all would agree that art, including literature, invites us to respond to it for its own sake (that is, non-instrumentally), some would argue that it demands this of us and that no instrumental use is legitimate or even possible. Neil Pickering, a philosopher qualified in literature, takes this sterner view, developing the claim in connection with the reading of poetry. For Pickering, to use poetry in order to address the purposes of an outside activity such as the education of doctors is to do something other than to read a poem, from which, he argues, it follows that the reading of poetry, and the use of poetry, are mutually exclusive.

Our two final substantive papers in this issue look at practical engagements between clinical medicine and the arts. Raimo Puustinen brings an interest in the nature of communication and in Bakhtin's theory of language to bear upon a home visit paid to an apparently depressive patient by the fictional Dr Koryolov, in Chekhov's $A$ Case History. A quite different problem is taken up by Søren Holm, when he examines the case for considering plastic and reconstructive surgery under the same aesthetic categories as the production of a work of acknowledged (plastic) art such as sculpture. Holm provocatively invites us to consider analogies with other body-altering practices, notably including bodybuilding, as a means to exploring the legitimate limits of what could count as "art" in clinical medicine.

The remainder of this issue is devoted to what are planned as regular features. Deborah Kirklin and Richard Meakin introduce a column on the humanities in medical education; Gillie Bolton presents a column which is devoted to discussing creative writing, including the exhibiting of original material in these pages. Ann Jay provides the first contribution to what is planned as a further regular feature, "Medicine through the novel". Readers interested in contributing to these columns are encouraged to contact the editors initially. Finally, book reviews and a short column of News and Notes complete what we hope will be found to be an informative, provocative and enjoyable first issue of this new journal.

\section{References}

1 Evans M, Greaves D. Exploring the medical humanities. British Medical fournal 1999; 319:1216.

2 Smith R. Editor's choice-struggling towards coherence. British Medical fournal 1999; 319.

3 Kopelman LM. Bioethics and humanities: what makes us a field? Fournal of Medicine and Philosophy 1998;23:356-68. 